\title{
Nasal NK/T cell lymphoma presents with long-term nasal blockage and fever: a rare case report and literature review
}

 \\ Zheng ${ }^{5,6}$ \\ ${ }^{1}$ Department of Infection Diseases, Zhejiang Provincial People's Hospital, Hangzhou, China \\ ${ }^{2}$ Department of Radiology, The First Affiliated Hospital of Wenzhou Medical University, Wenzhou, China \\ ${ }^{3}$ Department of Pathology, The First Affiliated Hospital of Wenzhou Medical University, Wenzhou, China \\ ${ }^{4}$ Department of Cardiovascular Medicine, Zhejiang Provincial People's Hospital, Hangzhou, China \\ ${ }^{5}$ Department of Infection and Liver Diseases, Liver Research Center, The First Affiliated Hospital of Wenzhou Medical \\ University, Wenzhou, China \\ ${ }^{6}$ Institute of Hepatology, Wenzhou Medical University, Wenzhou, China \\ Correspondence to: Ming-Hua Zheng, email: zhengmh@wmu.edu.cn \\ Ya-Hui Ding, email: dxh@163.com
}

Keywords: NK/T cell lymphoma, fever of unknown origin, clinical manifestation

Received: December 10,2015 Accepted: January 30, 2016

Published: February 14, 2016

\section{ABSTRACT}

NK/T cell lymphoma (NKTCL) is a common disease which is a threat to human health. Nasal NKTCL is a rare but serious type of systemic lymphoma because of its high mortality rate and serious complications. In this case report, we describe a male who presented with nasal blockage in the right side, a fever of one month duration and a soy-like, painless and gradually increasing mass in the right submandibular region due to nasal NKTCL. The patient had no significant medical history and the initial clinical symptoms were nasal blockage. Contrast computed tomography showed that the nasopharyngeal mucosa was thickened and that the celiac and retroperitoneal lymphaden was intumescent. Finally a biopsy, guided by nasal endoscopy and examined using flow cytometry confirmed a diagnosis of NKTCL. Nasal NKTCL is rare and has no unique characteristics at first presentation, such as epidemiology and obvious clinical manifestation. As no effective therapy is currently available for this disease, early diagnosis and therapy of nasal NKTCL remains challenging.

\section{INTRODUCTION}

NK/T cell lymphoma (NKTCL) is a common disease which threatens human health. NKTCL is a serious type of systemic lymphoma because of its high mortality rate and serious complications. In this case report, we describe a male who presented with nasal blockage in the right side, a fever of one month duration and a soylike, painless and gradually increasing mass in the right submandibular region due to NKTCL.

\section{CASE PRESENTATION}

A 21-year-old male was hospitalized with a tenmonth history of nasal blockage and a fever of one month duration. He presented with nasal blockage in the right side at the time of initial presentation, without nasal discharge, headache or tinnitus. Despite symptomatic treatment, he continued to do poorly and had persistent nasal blockage. He developed persistent fever a month ago, beginning at nightfall and peaking to $39{ }^{\circ} \mathrm{C}$ to 40 ${ }^{\circ} \mathrm{C}$ during the night or in the early morning. Meanwhile, a soy-like, painless and gradually increasing mass in the right submandibular area was found which was not responsive to antibiotic treatment. Fever improved when he received dexamethasone, and his temperature increased again when dexamethasone was withdrawn. Contrast computed tomography (CT) showed that the nasopharyngeal mucosa was thickened (Figure 1) and the celiac and retroperitoneal lymphaden was intumescent (Figure 2). Bone marrow aspiration and CT scan of chest and neck show normal. Finally, the biopsy based on nasal 


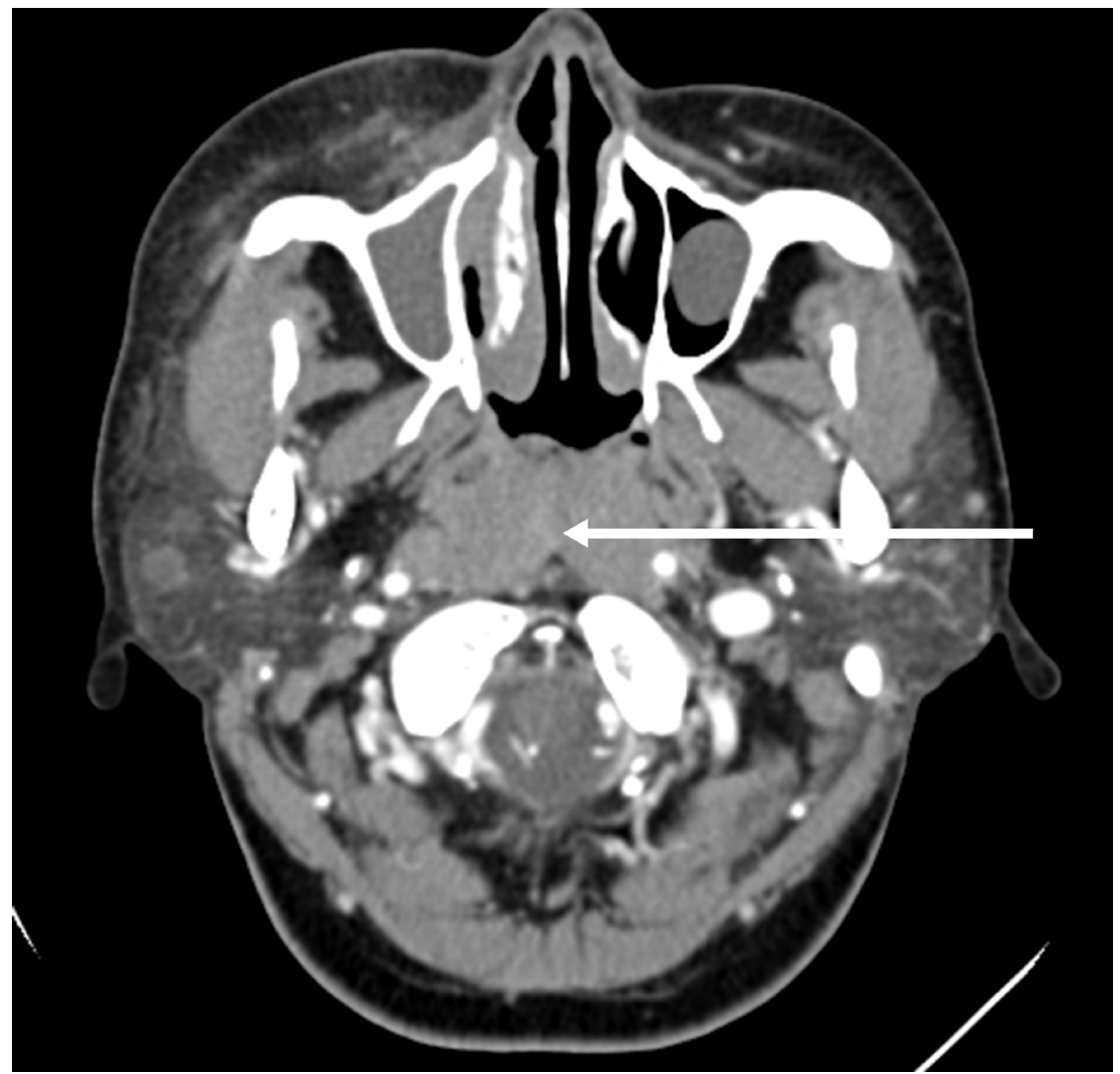

Figure 1: Contrast brain computed tomography shows diffuse swelling of the posterior wall of the pharynx with mild enhancement.

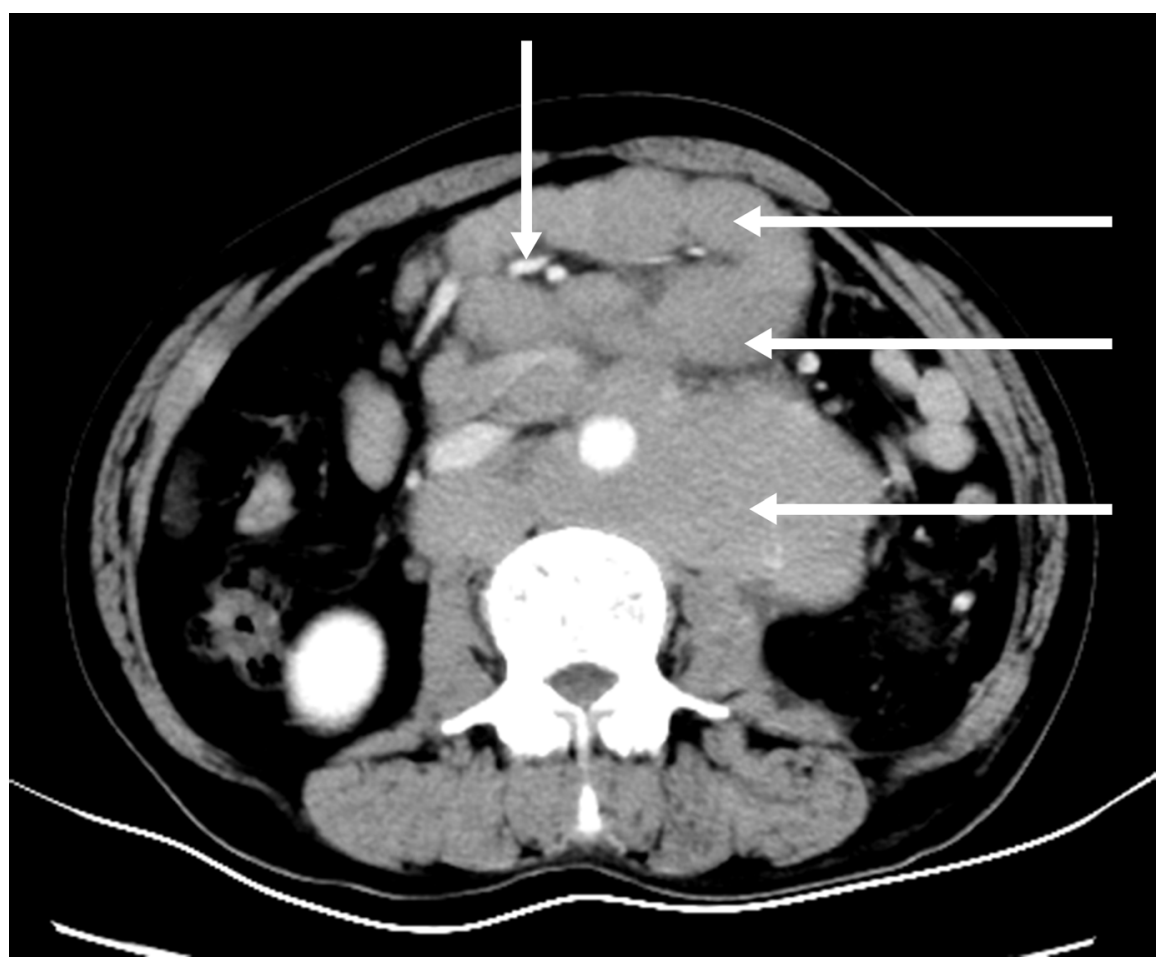

Figure 2: Contrast abdomen computed tomography shows multiple mesenteric and retroperitoneal lymph node enlargement and integration into masses with mild-middle enhancement, the mesenteric vessels embedded (sandwich sign). 
endoscopy and examination by flow cytometric methods confirmed a diagnosis of nasal NKTCL (Figure 3, Figure 4). According to the Ann Arbor staging, this patient was IIIB of the disease.

\section{DISCUSSION}

According to the WHO classification in 2001, nasal NKTCL as an independent lymphoma subtype mainly occurring in Asia, Mexico and South America [1], which could have a racial basis, however environmental factors also had to be considered [2].

Our patient first presented with nasal blockage, and the following computed tomography showed that the nasopharyngeal mucosa was thickened. Localized inflammation and tumor are the most likely diagnosis for this person. Nasopharyngitis and nasopharyngeal carcinoma (NPC) could cause thickening of nasopharyngeal mucosa, both of which are associated with viruses. NPC has a high incidence in southern China and
Hong Kong, with an incidence of 25 to 50 per 100,000 people [3]. Osteoma, papilloma, and carcinoma are relatively rare but are important potential causes and are responsible for tumors which may cause nasal blockage [4].The main symptoms are unilateral, slowly increasing blockage of the nose with or without bloody-purulent secretion and impairment of olfaction. In later stages of the disease, epistaxis and facial swelling can occur [5-6] and further progression can lead to ulceration and painful mid-facial destruction. The lymphoma could spread to the skin, gastrointestinal tract, testis, spleen and central nervous system [7]. However, nasal NKTCL could also lead to destruction and loss of function of the upper respiratory tract, especially in the nasal cavity, nasal and paranasal sinuses, and hard palate [8], which has been associated with Epstein barr virus infections [9].

Our patient has a soy-like, painless and gradually increasing mass in the right submandibular area, and has persistent fever beginning at nightfall and peaking during the night or in the early morning. Abdominal primary or metastatic malignant tumors often cause

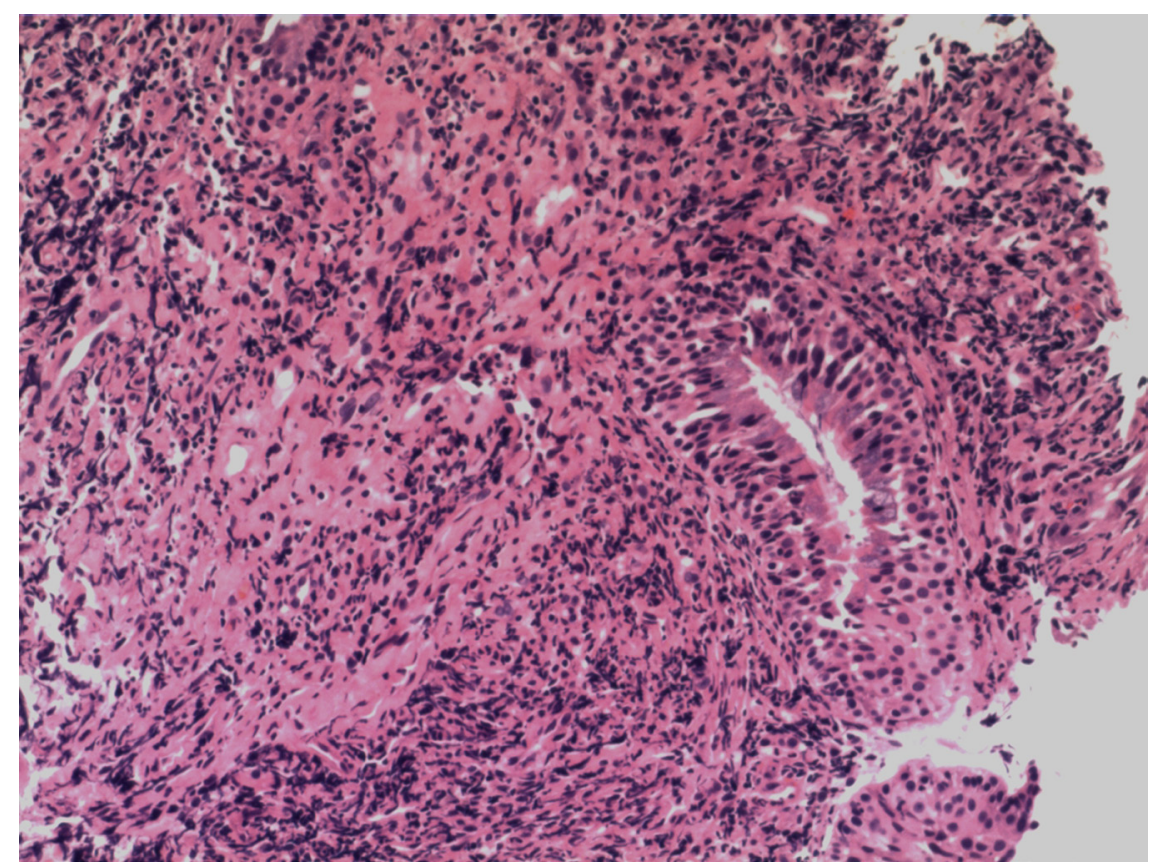

Figure 3: Pathology of the thickened nasopharyngeal mucosa
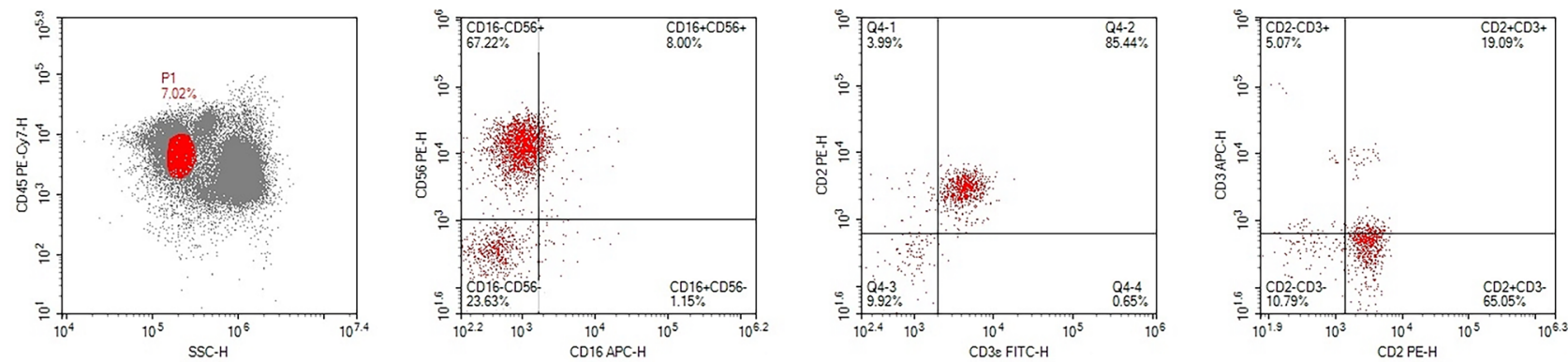

Figure 4: Result of the flow cytometry, based on the biopsy tissue 
abdominal lymphadenectasis, such as gastric, liver, and colorectal cancer. Mesenteric lymphadenitis can also cause abdominal lymphadenectasis which mainly occurs in children and is usually accompanied with secondary acute upper respiratory tract infection. A CT scan showed mesenteric and retroperitoneal lymphadenectasis. Tuberculous lymphadenitis could occur in the neck, armpits, abdomen and groin. In the early stage, patients commonly present with painless swollen lymph nodes. Some patients experience low-grade fever and night sweats. However, lymphoma is a common cause of lymphadenectasis, of which clinical symptoms are nonspecific, although fever is common. Infection, malignancy, collagenous vascular disease could cause fever of unknown origin (FUO). These diseases are usually hidden, and accompanied by atypical symptoms, such as subacute bacterial endocarditis, perforation of the retrocecal appendix, pericolonic abscess, occult liver or splenic abscesses, and prosthetic graft infections, which may easily be missed and eventually present as an undiagnosed prolonged fever [10-12].

However, cancer may account for $20 \%-30 \%$ of fever of FUO, of which lymphoma is the most common cause, accounting for $50 \%-70 \%$ of malignant tumors [13], where the initiating symptom is often lymphadenectasis. It should not be forgotten that because of the increase in the use of polypharmacy, the diagnosis of drug fever as a cause of FUO is relatively more common. Tumor was the most likely diagnosis for this person, but differential diagnoses need to consider inflammatory causes. Patients with long-term use of antibiotics, corticosteroids and immunosuppressants, uncontrolled diabetes mellitus, acquired immune deficiency syndrome are especially susceptible to fungal rhinosinusitis, which have different clinical features, including nasal blockage, runny nose, fever, and maxillary sinus mass. Aspergillus species, or members of the class zygomycetes are the most frequent etiological agents [14-18], which accounts for $80 \%$ of nasal symptoms.

Once confirmed as a nasal NKTCL, chemotherapy and radiation combination therapy is considered to be effective. The patient receives a total of 4 cycles of CHOP-L. After achieving complete remission with combined chemo-radiotherapy, hematopoietic stem cell transplantation is also recommended. Meanwhile, immunotherapy with pegylated interferon alpha during chemotherapy and radiation therapy may be also effective. International prognostic index score, near-term complete response rate [19], and other factors could be used to evaluate the prognosis [20].

\section{ACKNOWLEDGMENTS}

There was no financial support or funding for this case report. Informed patient consent was obtained for publication of this case report.

\section{CONFLICTS OF INTEREST}

The authors report no declarations of interest.

\section{REFERENCES}

1. You JY, Chi KH, Yang MH, Chen CC, Ho CH, Chau WK, Hsu HC, Gau JP, Tzeng CH, Liu JH, Chen PM, Chiou TJ. Radiation therapy versus chemotherapy as initial treatment for localized nasal natural killer (NK)/T-cell lymphoma: a single institute survey in Taiwan. Ann Oncol. 2004;15:618625 .

2. Aviles A. Nasal NK/T-Cell Lymphoma. A Comparative Analysis of a Mexican Population with the Other Populations of Latin-America. Mediterr J Hematol Infect Dis. 2015;7:e2015052.

3. Kamran SC, Riaz N, Lee N. Nasopharyngeal carcinoma. Surg Oncol Clin N Am. 2015;24:547-561.

4. Ledderose GJ, Berghaus A. Blocked nose: differential diagnosis [Article in German]. MMW Fortschr Med. 2015;157:41-44.

5. Rodrigo JP, Suarez C, Rinaldo A, Devaney KO, Carbone A, Barnes L, Heffner DK, Ferlito A. Idiopathic midline destructive disease: fact or fiction. Oral Oncol. 2005;41:340-348

6. Sheahan P, Donnelly M, O'Reilly S, Murphy M. T/NK cell non-Hodgkin's lymphoma of the sinonasal tract. J Laryngol Otol. 2001;115:1032-1035.

7. Kim GE, Koom WS, Yang WI, Lee SW, Keum KC, Lee CG, Suh CO, Hahn JS, Roh JK, Kim JH. Clinical relevance of three subtypes of primary sinonasal lymphoma characterized by immunophenotypic analysis. Head Neck. 2004;26:584-593.

8. William BM, Armitage JO. International analysis of the frequency and outcomes of NK/T-cell lymphomas. Best Pract Res Clin Haematol. 2013;26:23-32.

9. Kim WS, Song SY, Ahn YC, Ko YH, Baek CH, Kim DY, Yoon SS, Lee HG, Kang WK, Lee HJ, Park CH, Park K. CHOP followed by involved field radiation: is it optimal for localized nasal natural killer/T-cell lymphoma? Ann Oncol. 2001;12:349-352.

10. Brusch JL, Weinstein L. Fever of unknown origin. Med Clin North Am. 1988;72:1247-1261.

11. Cunha BA. Fever of unknown origin caused by adult juvenile rheumatoid arthritis: the diagnostic significance of double quotidian fevers and elevated serum ferritin levels. Heart Lung. 2004;33:417-421.

12. Bujak JS, Aptekar RG, Decker JL, Wolff SM. Juvenile rheumatoid arthritis presenting in the adult as fever of unknown origin. Medicine (Baltimore). 1973;52:431-444.

13. Saltoglu N, Tasova Y, Midikli D, Aksu HS, Sanli A, Dundar IH. Fever of unknown origin in Turkey: evaluation of 87 cases during a nine-year-period of study. J Infect. 2004;48:81-85. 
14. Ferguson BJ. Mucormycosis of the nose and paranasal sinuses. Otolaryngol Clin North Am. 2000;33:349-365.

15. Adelson RT, Marple BF. Fungal rhinosinusitis: state-of-theart diagnosis and treatment. J Otolaryngol. 2005;34 Suppl 1:S18-23.

16. Del Valle Zapico A, Rubio Suarez A, Mellado Encinas P, Morales Angulo C, Cabrera Pozuelo E. Mucormycosis of the sphenoid sinus in an otherwise healthy patient. Case report and literature review. J Laryngol Otol. 1996;110:471473.

17. Sridhara SR, Paragache G, Panda NK, Chakrabarti A. Mucormycosis in immunocompetent individuals: an increasing trend. J Otolaryngol. 2005;34:402-406.

18. Chakrabarti A, Das A, Mandal J, Shivaprakash MR, George VK, Tarai B, Rao P, Panda N, Verma SC, Sakhuja V. The rising trend of invasive zygomycosis in patients with uncontrolled diabetes mellitus. Med Mycol. 2006;44:335342.

19. Kim GE, Lee SW, Chang SK, Park HC, Pyo HR, Kim JH, Moon SR, Lee HS, Choi EC, Kim KM. Combined chemotherapy and radiation versus radiation alone in the management of localized angiocentric lymphoma of the head and neck. Radiother Oncol. 2001;61:261-269.

20. Kameoka Y, Takahashi N, Itou S, Kume M, Noji H, Kato Y, Ichikawa Y, Sasaki O, Motegi M, Ishiguro A, Tagawa H, Ishizawa K, Ishida $\mathrm{Y}$, et al. Analysis of clinical characteristics and prognostic factors for angioimmunoblastic T-cell lymphoma. Int $\mathrm{J}$ Hematol. 2015;101:536-542. 